\title{
Is Silybin the Best Free Radical Scavenger Compound in Silymarin?
}

\author{
Miguel Reina and Ana Martínez ${ }^{1}$ \\ Departamento de Materiales de Baja Dimensionalidad \\ Instituto de Investigaciones en Materiales \\ Universidad Nacional Autónoma de México \\ Circuito Exterior s/n, CU, P.O. Box 70-360, Coyoacán, 04510 \\ Ciudad de México, México \\ http://www.iim.unam.mx/martina/
}

Table S1. Relative abundance at $\mathrm{pH}=7.4$ for compounds under study

\begin{tabular}{cc} 
Molecule & Relative abundance at $\mathrm{pH}=7.4(\%)$ \\
\hline \hline SILYC $^{-1}$ & 68.76 \\
SILYC $_{(-\mathrm{H})}$ & 26.18 \\
\hline SIL $^{-1}$ & 69.08 \\
SIL $_{(-\mathrm{H})}$ & 26.30 \\
\hline ISOSIL $^{-1}$ & 69.08 \\
ISOSIL $_{(-\mathrm{H})}$ & 26.30 \\
\hline SILYD $^{-1}$ & 68.95 \\
SILYD $_{(-\mathrm{H})}$ & 26.22 \\
\hline TAX $^{-1}$ & 68.23 \\
TAX $_{(-\mathrm{H})}$ & 25.98 \\
\hline
\end{tabular}

\footnotetext{
${ }^{1}$ Corresponding author: martina@unam.mx
} 
Table S2. Dissociation energies (in $\mathrm{kcal} / \mathrm{mol}$ ) corresponding to the following reaction: $[\text { Silychristin-OOH }]^{\cdot} \rightarrow$ Silychristin $+{ }^{\circ} \mathrm{OOH}$.

The position where the free radical is bonded is as indicated in the schematic formula.

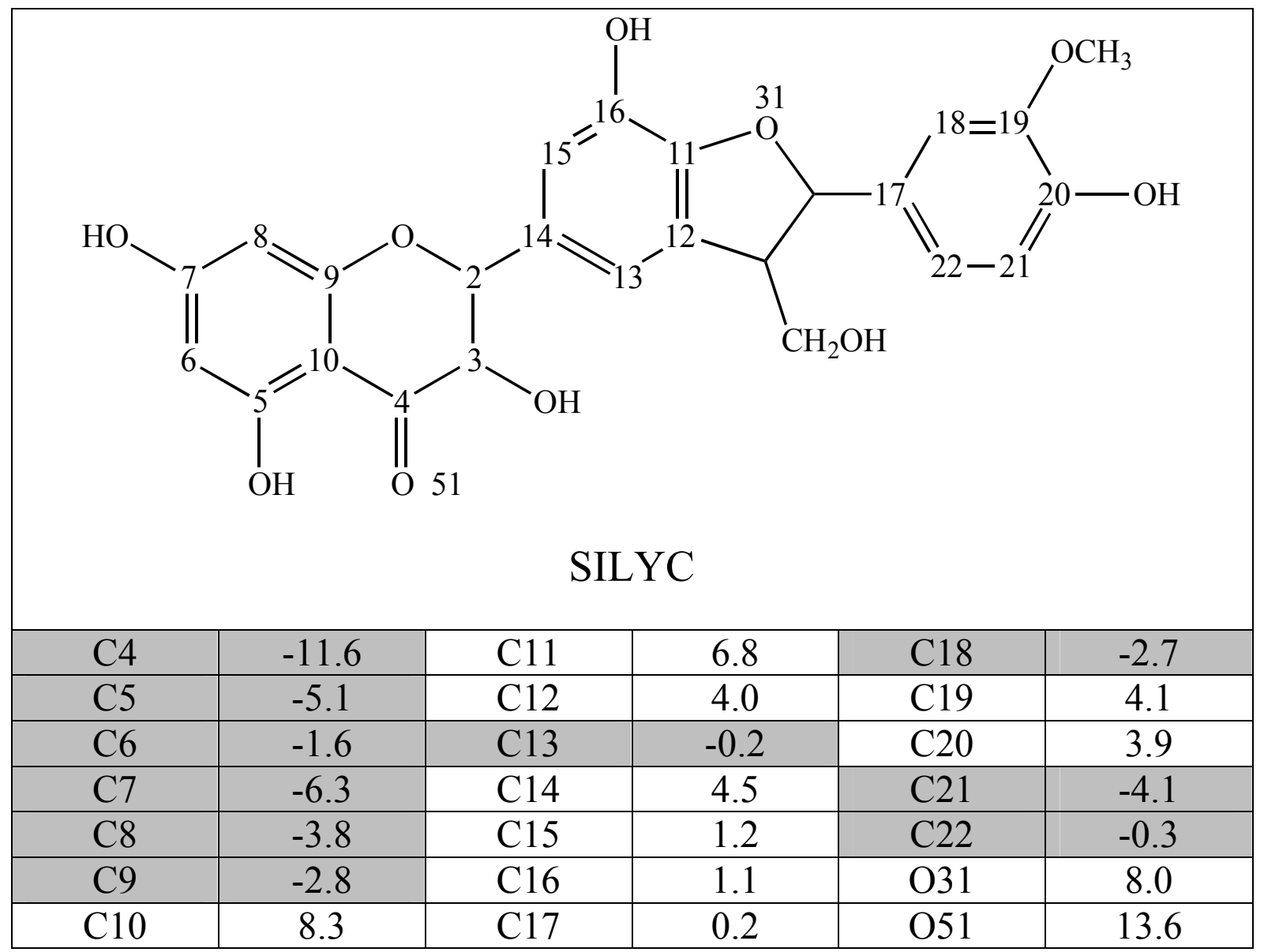


Table S3. Dissociation energies (in $\mathrm{kcal} / \mathrm{mol}$ ) corresponding to the following reaction: $[\text { Silybin-OOH }]^{\circ} \rightarrow$ Silybin $+{ }^{\circ} \mathrm{OOH}$.

The position where the free radical is bonded is as indicated in the schematic formula.

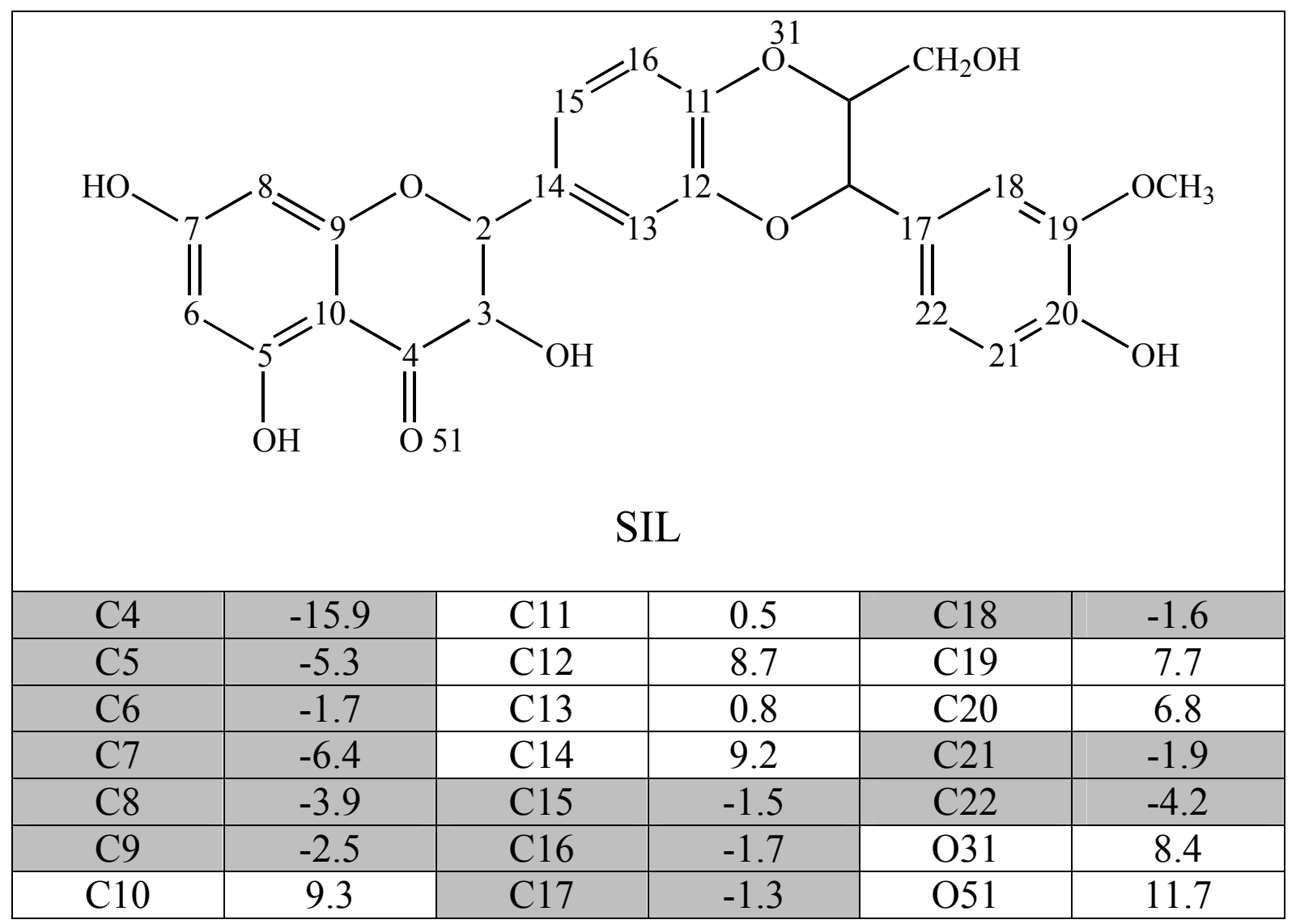


Table S4. Dissociation energies (in $\mathrm{kcal} / \mathrm{mol}$ ) corresponding to the following reaction: $[\text { Isosilybin-OOH }]^{\circ} \rightarrow$ Isosilybin $+{ }^{\circ} \mathrm{OOH}$.

The position where the free radical is bonded is as indicated in the schematic formula.

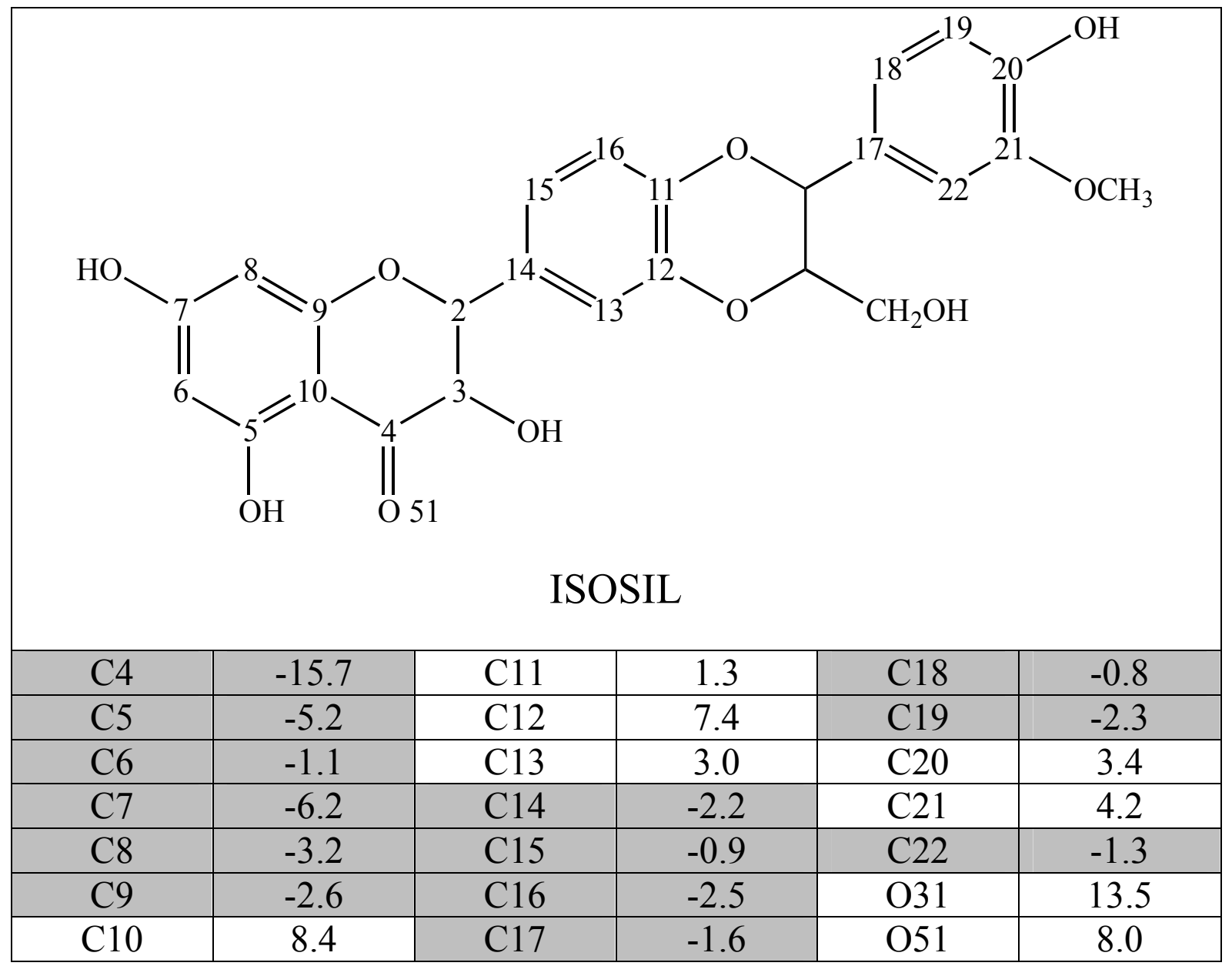


Table S5. Dissociation energies (in $\mathrm{kcal} / \mathrm{mol}$ ) corresponding to the following reaction: $[\text { Silydianin-OOH }]^{\bullet} \rightarrow$ Silydianin $+{ }^{\circ} \mathrm{OOH}$.

The position where the free radical is bonded is as indicated in the schematic formula

\begin{tabular}{|l|l|l|l|l|l|}
\hline & & \\
\\
\end{tabular}


Table S6. Dissociation energies (in $\mathrm{kcal} / \mathrm{mol}$ ) corresponding to the following reaction: $\left[\right.$ Taxifolin-OOH $^{\circ} \rightarrow$ Taxifolin $+{ }^{\circ} \mathrm{OOH}$.

The position where the free radical is bonded is as indicated in the schematic formula.

\begin{tabular}{|c|c|c|}
\hline \\
\\
\end{tabular}


Table S7. Dissociation energies (in $\mathrm{kcal} / \mathrm{mol}$ ) corresponding to the following reaction:

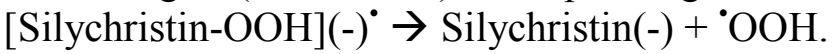

The position where the free radical is bonded is as indicated in the schematic formula.

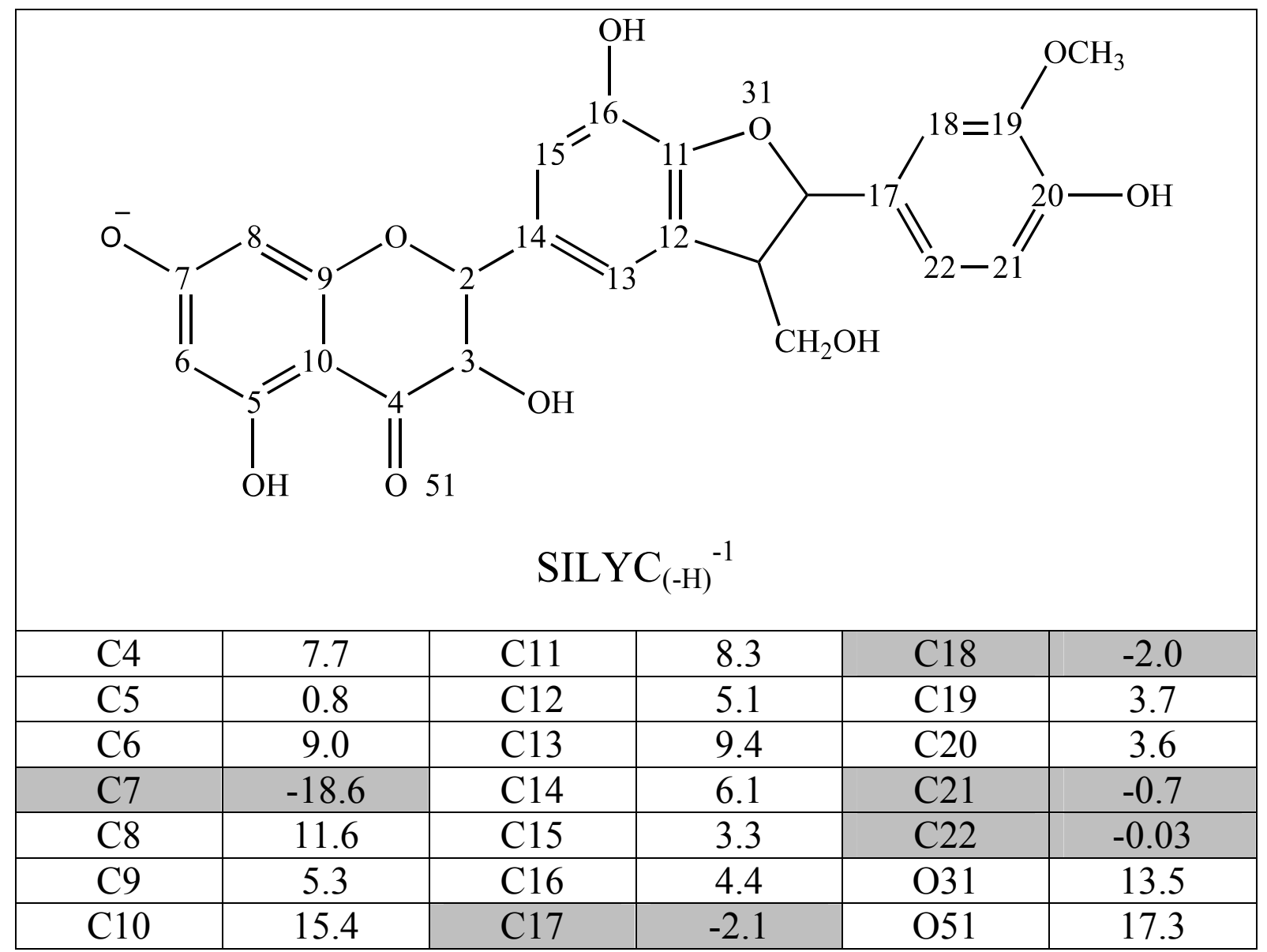


Table S8. Dissociation energies (in $\mathrm{kcal} / \mathrm{mol}$ ) corresponding to the following reaction: $\left[\right.$ Silybin-OOH $\left(^{-}\right)^{\bullet} \rightarrow$ Silybin(-) $+{ }^{\circ} \mathrm{OOH}$.

The position where the free radical is bonded is as indicated in the schematic formula.

\begin{tabular}{|c|c|c|c|c|c|}
\hline- & H & & $\begin{array}{c}31 \\
O\end{array}$ & & $\mathrm{OH}$ \\
\hline $\mathrm{C} 4$ & 12.5 & C11 & 2.4 & C18 & -3.3 \\
\hline C5 & 0.5 & C12 & 5.8 & C19 & 3.5 \\
\hline C6 & 9.3 & C13 & 3.4 & $\mathrm{C} 20$ & 3.7 \\
\hline C7 & -18.6 & C14 & -0.8 & C21 & -1.9 \\
\hline C8 & 12.1 & C15 & 3.4 & C22 & -5.2 \\
\hline C9 & 5.5 & $\mathrm{C} 16$ & -2.2 & O31 & 10.0 \\
\hline $\mathrm{C} 10$ & 12.4 & C17 & 1.7 & O51 & 17.6 \\
\hline
\end{tabular}


Table S9. Dissociation energies (in $\mathrm{kcal} / \mathrm{mol}$ ) corresponding to the following reaction: $[$ Isosilybin- $\mathrm{OOH}](-)^{\bullet} \rightarrow$ Isosilybin(-) $+{ }^{\circ} \mathrm{OOH}$.

The position where the free radical is bonded is as indicated in the schematic formula.

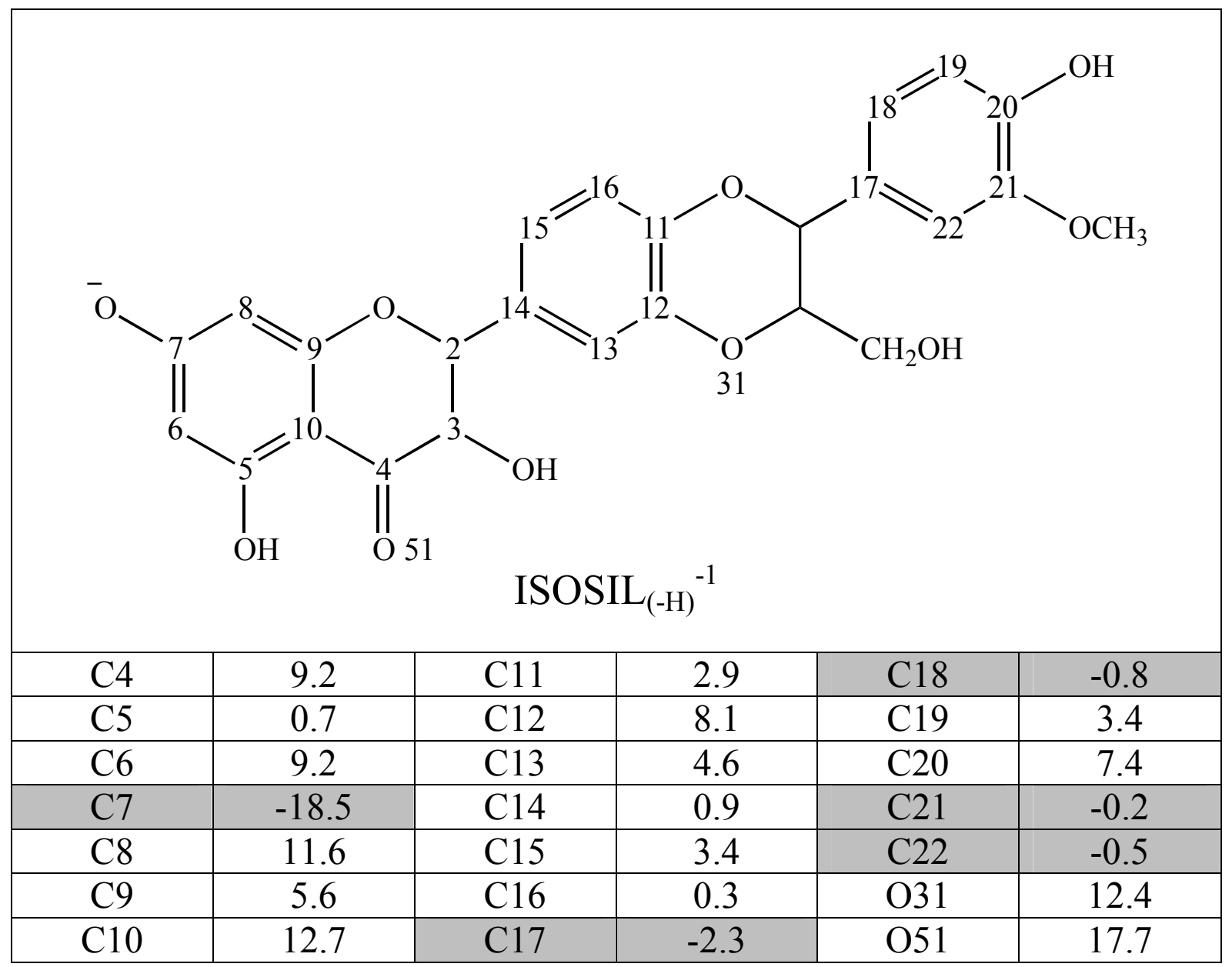


Table S10. Dissociation energies (in $\mathrm{kcal} / \mathrm{mol}$ ) corresponding to the following reaction: $[$ Silydianin-OOH $](-)^{\bullet} \rightarrow$ Silydianin $(-)+{ }^{\circ} \mathrm{OOH}$.

The position where the free radical is bonded is as indicated in the schematic formula.

\begin{tabular}{|l|l|l|l|l|l|}
\hline & & & \\
\\
\end{tabular}


Table S11. Dissociation energies (in $\mathrm{kcal} / \mathrm{mol}$ ) corresponding to the following reaction: $[$ Taxifolin-OOH $](-)^{\circ} \rightarrow$ Taxifolin(-) + ${ }^{\circ} \mathrm{OOH}$.

The position where the free radical is bonded is as indicated in the schematic formula.

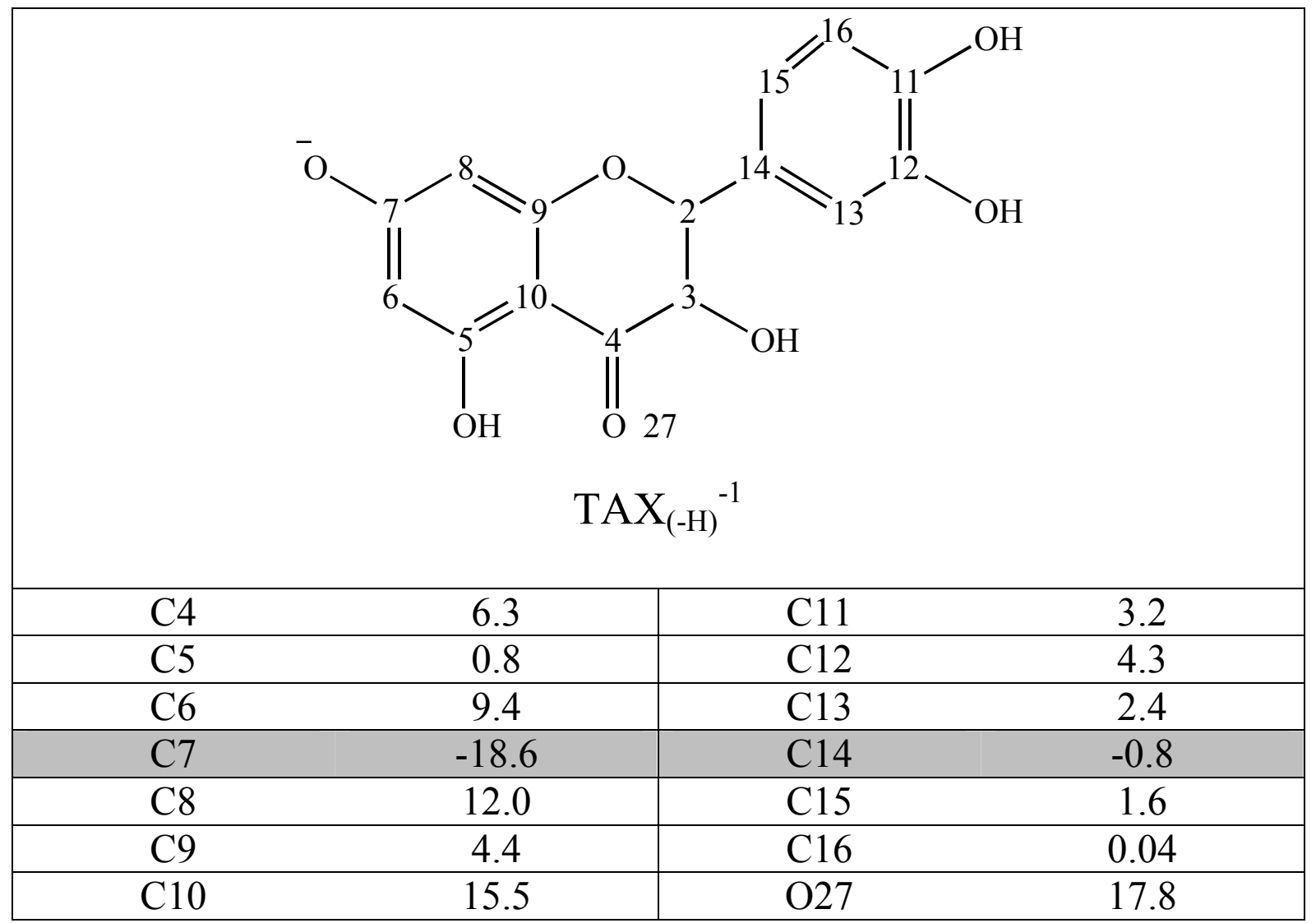


References with more than 10 authors

17.- Trouillas, P.; Marsal, P.; Svobodová, A.; Vostálová, J.; Gazak, R.; Hrbác, J.; Sedmera, P.; Kren, V.; Lazzaroni, R.; Duroux, J.L.; Walterová, D. Mechanism of the antioxidant action of silybin and 2,3-dehydrosilybin flavonolignans: A Joint Experimental and Theoretical Study. J. Phys. Chem. A. 2008, 112, 1054-1063.

27.- Gaussian 09, Revision A.08, Frisch, M. J.; Trucks, G. W.; Schlegel, H. B.; Scuseria, G. E.; Robb, M. A.; Cheeseman, J. R.; Scalmani, G.; Barone, V.; Mennucci, B.; Petersson, G. A.; Nakatsuji, H.; Caricato, M.; Li, X.; Hratchian, H. P.; Izmaylov, A. F.; Bloino, J.; Zheng, G.; Sonnenberg, J. L.; Hada, M.; Ehara, M.; Toyota, K.; Fukuda, R.; Hasegawa, J.; Ishida, M.; Nakajima, T.; Honda, Y.; Kitao, O.; Nakai, H.; Vreven, T.; Montgomery, J. A. Jr.; Peralta, J. E.; Ogliaro, F.; Bearpark, M.; Heyd, J. J.; Brothers, E.; Kudin, K. N.; Staroverov, V. N.; Kobayashi, R.; Normand, J.; Raghavachari, K.; Rendell, A.; Burant, J. C.; Iyengar, S. S.; Tomasi, J.; Cossi, M. R.; Millam, N. J.; Klene, M.; Knox, J. E.; Cross, J. B.; Bakken, V.; Adamo, C.; Jaramillo, J.; Gomperts, R. E.; Stratmann, O.; Yazyev, A. J.; Austin, R.; Cammi, C.; Pomelli, J. W.; Ochterski, R.; Martin, R. L.; Morokuma, K.; Zakrzewski, V. G.; Voth, G. A.; Salvador, P.; Dannenberg, J. J.; Dapprich, S.; Daniels, A. D.; Farkas, O.; Foresman, J. B.; Ortiz, J. V.; Cioslowski, J.; Fox, D. J. Gaussian, Inc., Wallingford CT, 2009. 


\section{CARTESIAN COORDINATES OF THE OPTIMIZED STRUCTURES}

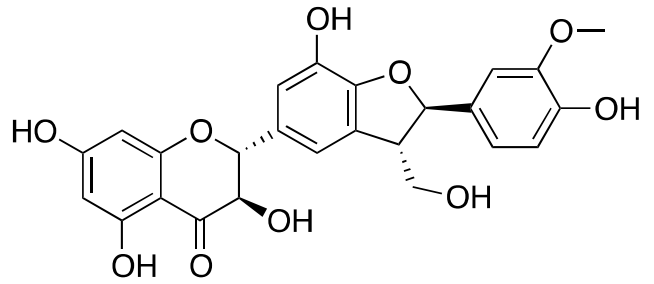

SILYC

\begin{tabular}{|c|c|c|c|c|c|c|}
\hline \multirow{2}{*}{$\begin{array}{l}\text { Center } \\
\text { Number } \\
\text {------ }\end{array}$} & \multirow{2}{*}{$\begin{array}{l}\text { Atomic } \\
\text { Number }\end{array}$} & \multirow{2}{*}{\multicolumn{2}{|c|}{$\begin{array}{l}\text { Atomic } \\
\text { Type }\end{array}$}} & \multicolumn{3}{|c|}{ Coordinates (Angstroms) } \\
\hline & & & & $\mathrm{X}$ & $\mathrm{Y}$ & $\mathrm{Z}$ \\
\hline 1 & & 6 & 0 & -7.650609 & -0.234583 & -0.205277 \\
\hline 2 & & 6 & 0 & -6.785715 & 0.846156 & -0.098544 \\
\hline 3 & & 6 & 0 & -5.386207 & 0.635512 & 0.021556 \\
\hline 4 & & 6 & 0 & -4.893996 & -0.694131 & -0.007364 \\
\hline 5 & & 6 & 0 & -5.750279 & -1.773892 & -0.112029 \\
\hline 6 & & 6 & 0 & -7.12314 & -1.52709 & -0.202838 \\
\hline 7 & & 1 & 0 & -8.721095 & -0.051375 & -0.288047 \\
\hline 8 & & 1 & 0 & -5.365399 & -2.789294 & -0.141007 \\
\hline 9 & & 8 & 0 & -7.300965 & 2.076487 & -0.093327 \\
\hline 10 & & 1 & 0 & -6.560527 & 2.724979 & -0.0116 \\
\hline 11 & & 8 & 0 & -7.919689 & -2.611392 & -0.30763 \\
\hline 12 & & 1 & 0 & -8.846226 & -2.338006 & -0.377979 \\
\hline 13 & & 8 & 0 & -3.573013 & -0.951881 & 0.077462 \\
\hline 14 & & 6 & 0 & -4.480593 & 1.724117 & 0.237526 \\
\hline 15 & & 8 & 0 & -4.797028 & 2.923252 & 0.212107 \\
\hline 16 & & 6 & 0 & -3.044781 & 1.352363 & 0.539775 \\
\hline 17 & & 1 & 0 & -2.997003 & 1.085884 & 1.617425 \\
\hline 18 & & 8 & 0 & -2.184499 & 2.40614 & 0.241339 \\
\hline 19 & & 1 & 0 & -2.733341 & 3.212438 & 0.228966 \\
\hline 20 & & 6 & 0 & -2.665284 & 0.112176 & -0.263599 \\
\hline 21 & & 1 & 0 & -2.791018 & 0.345487 & -1.33607 \\
\hline 22 & & 6 & 0 & -1.265765 & -0.35393 & -0.007933 \\
\hline 23 & & 6 & 0 & -0.948302 & -0.953941 & 1.215414 \\
\hline 24 & & 6 & 0 & -0.281443 & -0.133969 & -0.975025 \\
\hline 25 & & 6 & 0 & 0.354354 & -1.347941 & 1.496989 \\
\hline 26 & & 1 & 0 & -1.719581 & -1.145943 & 1.960596 \\
\hline 27 & & 6 & 0 & 1.019464 & -0.529058 & -0.69647 \\
\hline 28 & & 1 & 0 & -0.525063 & 0.355624 & -1.916785 \\
\hline 29 & & 6 & 0 & 1.315996 & -1.114225 & 0.521837 \\
\hline
\end{tabular}




$\begin{array}{rrrrrr}30 & 8 & 0 & 2.63365 & -1.434159 & 0.683886 \\ 31 & 6 & 0 & 3.232646 & -1.340525 & -0.62983 \\ 32 & 6 & 0 & 2.318439 & -0.385413 & -1.445762 \\ 33 & 1 & 0 & 2.249526 & -0.737052 & -2.486228 \\ 34 & 6 & 0 & 2.784833 & 1.062549 & -1.488106 \\ 35 & 1 & 0 & 3.81043 & 1.119149 & -1.891228 \\ 36 & 1 & 0 & 2.796842 & 1.481912 & -0.465678 \\ 37 & 8 & 0 & 1.874116 & 1.752997 & -2.319083 \\ 38 & 1 & 0 & 2.02668 & 2.703807 & -2.247378 \\ 39 & 6 & 0 & 4.675292 & -0.9562 & -0.517883 \\ 40 & 6 & 0 & 5.16016 & -0.210208 & 0.553238 \\ 41 & 6 & 0 & 5.558373 & -1.306328 & -1.5411 \\ 42 & 6 & 0 & 6.490335 & 0.20167 & 0.6136 \\ 43 & 1 & 0 & 4.480707 & 0.055817 & 1.36447 \\ 44 & 6 & 0 & 6.889695 & -0.918493 & -1.497932 \\ 45 & 1 & 0 & 5.199013 & -1.90185 & -2.382733 \\ 46 & 6 & 0 & 7.36391 & -0.169566 & -0.42113 \\ 47 & 1 & 0 & 7.591441 & -1.199722 & -2.280717 \\ 48 & 1 & 0 & 3.153521 & -2.344819 & -1.080645 \\ 49 & 8 & 0 & 8.670877 & 0.186618 & -0.425198 \\ 50 & 1 & 0 & 8.934159 & 0.402289 & 0.491264 \\ 51 & 8 & 0 & 0.655868 & -1.935398 & 2.684478 \\ 52 & 1 & 0 & 1.591442 & -2.191797 & 2.678525 \\ 53 & 8 & 0 & 6.943555 & 1.035857 & 1.683003 \\ 54 & 6 & 0 & 6.388196 & 2.306986 & 2.030435 \\ 55 & 1 & 0 & 6.05114 & 2.803154 & 1.14437 \\ 56 & 1 & 0 & 5.562449 & 2.165532 & 2.696043 \\ 57 & 1 & 0 & 7.13545 & 2.903395 & 2.510859 \\ ----- & & ------------ & ------------ & -----------\end{array}$


<smiles>COc1cc(C2Oc3cc([C@H]4Oc5cc(O)cc(O)c5C(=O)[C@H]4O)ccc3OC2CO)ccc1O</smiles>

SIL

\begin{tabular}{|c|c|c|c|c|c|}
\hline \multirow{2}{*}{$\begin{array}{l}\text { Center } \\
\text { Number }\end{array}$} & \multirow{2}{*}{$\begin{array}{l}\text { Atomic } \\
\text { Number }\end{array}$} & Atomic & \multicolumn{3}{|c|}{ Coordinates (Angstroms) } \\
\hline & & Type & $\mathrm{X}$ & Y & Z \\
\hline 1 & 6 & 0 & 1525 & 0041032 & -1124107 \\
\hline 1 & 6 & 0 & -6917561 & 0.041053 & -1.124101 \\
\hline 3 & 6 & 0 & -5.578732 & 0.659134 & -0.163083 \\
\hline 4 & 6 & 0 & -5.087093 & -0.631558 & -0.490637 \\
\hline 5 & 6 & 0 & -5.892843 & -1.573232 & -1.101243 \\
\hline 6 & 6 & 0 & -7.212178 & -1.223737 & -1.403933 \\
\hline 7 & 1 & 0 & -8.757294 & 0.308886 & -1.373849 \\
\hline 8 & 1 & 0 & -5.506976 & -2.555502 & -1.358623 \\
\hline 9 & 8 & 0 & -7.427382 & 2.185164 & -0.238809 \\
\hline 10 & 1 & 0 & -6.728193 & 2.73132 & 0.195494 \\
\hline 11 & 8 & 0 & -7.959049 & -2.17479 & -2.001963 \\
\hline 12 & 1 & 0 & -8.847979 & -1.835655 & -2.183912 \\
\hline 13 & 8 & 0 & -3.816443 & -0.988958 & -0.208634 \\
\hline 14 & 6 & 0 & -4.749612 & 1.579911 & 0.558442 \\
\hline 15 & 8 & 0 & -5.060802 & 2.749611 & 0.828186 \\
\hline 16 & 6 & 0 & -3.412053 & 1.051742 & 1.030881 \\
\hline 17 & 1 & 0 & -3.593325 & 0.484344 & 1.969177 \\
\hline 18 & 8 & 0 & -2.508594 & 2.091842 & 1.234657 \\
\hline 19 & 1 & 0 & -3.041338 & 2.898775 & 1.362281 \\
\hline 20 & 6 & 0 & -2.870524 & 0.069562 & -0.001067 \\
\hline 21 & 1 & 0 & -2.756073 & 0.631006 & -0.946213 \\
\hline 22 & 6 & 0 & -1.557719 & -0.530779 & 0.398352 \\
\hline 23 & 6 & 0 & -1.501067 & -1.637923 & 1.249407 \\
\hline 24 & 6 & 0 & -0.375894 & 0.031515 & -0.071845 \\
\hline 25 & 6 & 0 & -0.2748 & -2.16323 & 1.631799 \\
\hline 26 & 1 & 0 & -2.422084 & -2.101712 & 1.599045 \\
\hline 27 & 6 & 0 & 0.852332 & -0.490363 & 0.31379 \\
\hline 28 & 1 & 0 & -0.386386 & 0.890932 & -0.741903 \\
\hline 29 & 6 & 0 & 0.907278 & -1.58275 & 1.18039 \\
\hline 30 & 8 & 0 & 1.991267 & 0.096413 & -0.158641 \\
\hline 31 & 8 & 0 & 2.094647 & -2.112938 & 1.582969 \\
\hline 32 & 6 & 0 & 3.239094 & -1.298126 & 1.331863 \\
\hline
\end{tabular}




\begin{tabular}{|c|c|c|c|c|c|}
\hline 33 & 1 & 0 & 4.095634 & -1.983941 & 1.387418 \\
\hline 34 & 6 & 0 & 3.154957 & -0.718621 & -0.08611 \\
\hline 35 & 1 & 0 & 3.039902 & -1.549419 & -0.807328 \\
\hline 36 & 6 & 0 & 3.391458 & -0.26189 & 2.445307 \\
\hline 37 & 1 & 0 & 3.078022 & -0.738259 & 3.381856 \\
\hline 38 & 1 & 0 & 2.726653 & 0.601478 & 2.272577 \\
\hline 39 & 8 & 0 & 4.731577 & 0.129636 & 2.633956 \\
\hline 40 & 1 & 0 & 4.989226 & 0.729083 & 1.916133 \\
\hline 41 & 6 & 0 & 4.351545 & 0.112211 & -0.453096 \\
\hline 42 & 6 & 0 & 4.340768 & 1.500465 & -0.365492 \\
\hline 43 & 6 & 0 & 5.516295 & -0.544839 & -0.872772 \\
\hline 44 & 6 & 0 & 5.491111 & 2.234233 & -0.664311 \\
\hline 45 & 1 & 0 & 3.4283 & 2.013936 & -0.069893 \\
\hline 46 & 6 & 0 & 6.659244 & 0.183349 & -1.164261 \\
\hline 47 & 1 & 0 & 5.516549 & -1.631439 & -0.959188 \\
\hline 48 & 6 & 0 & 6.650097 & 1.586259 & -1.056967 \\
\hline 49 & 8 & 0 & 7.761166 & 2.301252 & -1.347549 \\
\hline 50 & 1 & 0 & -0.205472 & -3.023699 & 2.294986 \\
\hline 51 & 1 & 0 & 5.500235 & 3.320688 & -0.601995 \\
\hline 52 & 1 & 0 & 8.467139 & 1.682544 & -1.599033 \\
\hline 53 & 8 & 0 & 7.851689 & -0.332288 & -1.57498 \\
\hline 54 & 6 & 0 & 7.960598 & -1.733795 & -1.698891 \\
\hline 55 & 1 & 0 & 8.985281 & -1.935877 & -2.018951 \\
\hline 56 & 1 & 0 & 7.77401 & -2.229352 & -0.735453 \\
\hline 57 & 1 & 0 & 7.260554 & -2.11845 & -2.454424 \\
\hline
\end{tabular}


<smiles>COc1cc([C@H]2Oc3ccc([C@@H]4Oc5cc(O)cc(O)c5C(=O)[C@H](O)[C@H]4CO)cc3O[C@H]2CO)ccc1O</smiles>

ISOSIL

\begin{tabular}{|c|c|c|c|c|c|}
\hline \multirow{2}{*}{$\begin{array}{l}\text { Center } \\
\text { Number }\end{array}$} & \multirow{2}{*}{$\begin{array}{l}\text { Atomic } \\
\text { Number }\end{array}$} & \multirow{2}{*}{$\begin{array}{l}\text { Atomic } \\
\text { Type }\end{array}$} & \multicolumn{3}{|c|}{ Coordinates (Angstroms) } \\
\hline & & & $\mathrm{X}$ & $\mathrm{Y}$ & $\mathrm{Z}$ \\
\hline 1 & 6 & 0 & 8.045047 & -0.694303 & 0.400499 \\
\hline 2 & 6 & 0 & 7.308518 & 0.343094 & -0.17781 \\
\hline 3 & 6 & 0 & 5.926635 & 0.198838 & -0.347401 \\
\hline 4 & 6 & 0 & 5.283402 & -0.986342 & 0.064052 \\
\hline 5 & 6 & 0 & 6.005222 & -1.991797 & 0.625378 \\
\hline 6 & 6 & 0 & 7.391264 & -1.864267 & 0.802664 \\
\hline 7 & 1 & 0 & 9.101887 & -0.594591 & 0.534833 \\
\hline 8 & 1 & 0 & 5.511797 & -2.889283 & 0.935135 \\
\hline 9 & 8 & 0 & 7.903851 & 1.43655 & -0.556378 \\
\hline 10 & 1 & 0 & 7.395117 & 2.147342 & -0.953322 \\
\hline 11 & 8 & 0 & 8.136604 & -2.931237 & 1.395069 \\
\hline 12 & 1 & 0 & 9.030517 & -2.930007 & 1.045043 \\
\hline 13 & 8 & 0 & 3.86306 & -1.155005 & -0.101347 \\
\hline 14 & 6 & 0 & 5.184356 & 1.232754 & -0.925396 \\
\hline 15 & 8 & 0 & 5.74412 & 2.298867 & -1.29096 \\
\hline 16 & 6 & 0 & 3.676532 & 1.041681 & -1.10232 \\
\hline 17 & 1 & 0 & 3.477812 & 0.610718 & -2.06132 \\
\hline 18 & 8 & 0 & 3.010715 & 2.303394 & -1.004003 \\
\hline 19 & 1 & 0 & 3.332673 & 2.889639 & -1.692667 \\
\hline 20 & 6 & 0 & 3.177021 & 0.09924 & 0.001454 \\
\hline 21 & 1 & 0 & 3.371918 & 0.534429 & 0.95933 \\
\hline 22 & 6 & 0 & 1.662145 & -0.125248 & -0.160892 \\
\hline 23 & 6 & 0 & 1.191876 & -1.177975 & -0.952239 \\
\hline 24 & 6 & 0 & 0.753808 & 0.727773 & 0.482208 \\
\hline 25 & 6 & 0 & -0.184382 & -1.391615 & -1.067663 \\
\hline 26 & 1 & 0 & 1.881026 & -1.816993 & -1.463734 \\
\hline 27 & 6 & 0 & -0.585487 & 0.542061 & 0.345514 \\
\hline 28 & 1 & 0 & 1.118523 & 1.535394 & 1.0819 \\
\hline 29 & 6 & 0 & -1.076968 & -0.550385 & -0.391577 \\
\hline
\end{tabular}




\begin{tabular}{|c|c|c|c|c|c|}
\hline 30 & 8 & 0 & -1.479245 & 1.482839 & 0.948625 \\
\hline 31 & 8 & 0 & -2.478798 & -0.830218 & -0.451445 \\
\hline 32 & 1 & 0 & -0.554502 & -2.197751 & -1.666041 \\
\hline 33 & 6 & 0 & -3.280706 & 0.316615 & -0.157513 \\
\hline 34 & 1 & 0 & -3.207431 & 1.038897 & -0.94354 \\
\hline 35 & 6 & 0 & -2.787871 & 0.944402 & 1.155551 \\
\hline 36 & 1 & 0 & -2.770581 & 0.196008 & 1.920082 \\
\hline 37 & 6 & 0 & -3.729652 & 2.086802 & 1.579311 \\
\hline 38 & 1 & 0 & -4.711925 & 1.69628 & 1.745238 \\
\hline 39 & 1 & 0 & -3.765352 & 2.825137 & 0.805691 \\
\hline 40 & 8 & 0 & -3.243692 & 2.682769 & 2.784951 \\
\hline 41 & 1 & 0 & -3.957387 & 3.156156 & 3.218707 \\
\hline 42 & 6 & 0 & -4.746639 & -0.131498 & -0.009747 \\
\hline 43 & 6 & 0 & -5.729883 & 0.416004 & -0.834335 \\
\hline 44 & 6 & 0 & -5.091409 & -1.084776 & 0.948346 \\
\hline 45 & 6 & 0 & -7.057678 & 0.010756 & -0.700334 \\
\hline 46 & 1 & 0 & -5.45787 & 1.168095 & -1.58906 \\
\hline 47 & 6 & 0 & -6.419409 & -1.49098 & 1.08185 \\
\hline 48 & 1 & 0 & -4.316558 & -1.516526 & 1.598212 \\
\hline 49 & 6 & 0 & -7.402546 & -0.943306 & 0.257816 \\
\hline 50 & 1 & 0 & -6.690886 & -2.242918 & 1.837074 \\
\hline 51 & 8 & 0 & -8.764009 & -1.358661 & 0.394916 \\
\hline 52 & 1 & 0 & -9.057365 & -0.504832 & 0.068543 \\
\hline 53 & 8 & 0 & -8.370983 & 0.215876 & -1.227645 \\
\hline 54 & 6 & 0 & -8.785899 & -0.950961 & -1.942653 \\
\hline 55 & 1 & 0 & -9.565811 & -1.443192 & -1.400115 \\
\hline 56 & 1 & 0 & -9.147703 & -0.667346 & -2.908862 \\
\hline 57 & 1 & 0 & -7.954646 & -1.615433 & -2.053986 \\
\hline
\end{tabular}




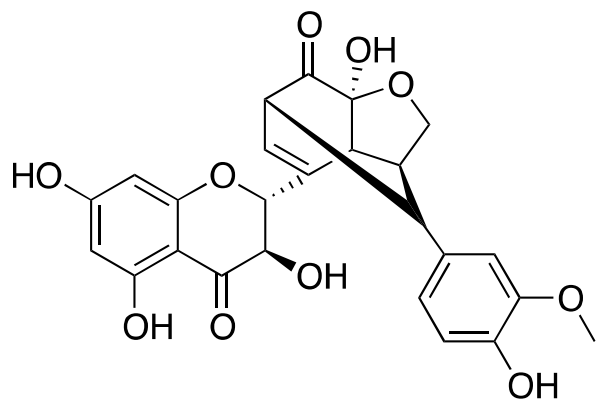

SILYD

\begin{tabular}{|c|c|c|c|c|c|}
\hline \multirow{2}{*}{$\begin{array}{l}\text { Center } \\
\text { Number }\end{array}$} & \multirow{2}{*}{$\begin{array}{l}\text { Atomic } \\
\text { Number }\end{array}$} & \multirow{2}{*}{$\begin{array}{l}\text { Atomic } \\
\text { Type }\end{array}$} & \multicolumn{3}{|c|}{ Coordinates (Angstroms) } \\
\hline & & & $\mathrm{X}$ & $\mathrm{Y}$ & Z \\
\hline 1 & 6 & 0 & -1.115354 & -1.851932 & 0.386749 \\
\hline 2 & 8 & 0 & -2.33803 & -2.301073 & -0.226759 \\
\hline 3 & 6 & 0 & -2.491696 & -1.638303 & -1.497158 \\
\hline 4 & 6 & 0 & -1.53416 & -0.446492 & -1.441226 \\
\hline 5 & 6 & 0 & -0.322429 & -1.124599 & -0.724504 \\
\hline 6 & 1 & 0 & -2.207131 & -2.330231 & -2.301622 \\
\hline 7 & 1 & 0 & -3.541249 & -1.362753 & -1.617945 \\
\hline 8 & 1 & 0 & -1.281747 & -0.097511 & -2.447375 \\
\hline 9 & 1 & 0 & 0.162711 & -1.859958 & -1.367482 \\
\hline 10 & 6 & 0 & 0.647769 & -0.140699 & -0.107549 \\
\hline 11 & 6 & 0 & 0.083735 & 0.739646 & 0.73706 \\
\hline 12 & 1 & 0 & 0.636769 & 1.494193 & 1.284258 \\
\hline 13 & 6 & 0 & -1.423478 & 0.617799 & 0.880518 \\
\hline 14 & 1 & 0 & -1.846471 & 1.34439 & 1.576404 \\
\hline 15 & 6 & 0 & -1.559914 & -0.798339 & 1.426417 \\
\hline 16 & 8 & 0 & -0.427985 & -2.894564 & 0.975268 \\
\hline 17 & 1 & 0 & -0.845989 & -3.061458 & 1.841557 \\
\hline 18 & 8 & 0 & -1.918235 & -1.108709 & 2.547945 \\
\hline 19 & 6 & 0 & -2.032572 & 0.761075 & -0.562726 \\
\hline 20 & 1 & 0 & -1.547942 & 1.652991 & -0.975255 \\
\hline 21 & 6 & 0 & -3.52784 & 1.030906 & -0.599837 \\
\hline 22 & 6 & 0 & -4.036844 & 1.942464 & -1.531741 \\
\hline 23 & 6 & 0 & -4.430624 & 0.391635 & 0.271579 \\
\hline 24 & 6 & 0 & -5.407573 & 2.212911 & -1.612694 \\
\hline 25 & 1 & 0 & -3.357009 & 2.458942 & -2.205901 \\
\hline 26 & 6 & 0 & -5.795136 & 0.661607 & 0.198992 \\
\hline 27 & 1 & 0 & -4.069282 & -0.318692 & 1.005238 \\
\hline 28 & 6 & 0 & -6.292273 & 1.576604 & -0.75063 \\
\hline 29 & 1 & 0 & -5.799524 & 2.922858 & -2.335083 \\
\hline 30 & 8 & 0 & -7.629812 & 1.841693 & -0.819799 \\
\hline
\end{tabular}




$\begin{array}{rrrrrr}31 & 1 & 0 & -8.075895 & 1.305993 & -0.140169 \\ 32 & 8 & 0 & -6.764568 & 0.103249 & 0.995772 \\ 33 & 6 & 0 & -6.371749 & -0.848471 & 1.983669 \\ 34 & 1 & 0 & -5.689452 & -0.397527 & 2.714059 \\ 35 & 1 & 0 & -7.29286 & -1.155496 & 2.481361 \\ 36 & 1 & 0 & -5.892851 & -1.719443 & 1.520662 \\ 37 & 6 & 0 & 2.114825 & -0.155989 & -0.46313 \\ 38 & 6 & 0 & 2.834438 & -1.499414 & -0.22251 \\ 39 & 6 & 0 & 4.341098 & -1.336332 & -0.428156 \\ 40 & 6 & 0 & 4.093176 & 1.021548 & 0.258001 \\ 41 & 6 & 0 & 4.924919 & -0.063061 & -0.127077 \\ 42 & 8 & 0 & 2.344847 & -2.473942 & -1.116941 \\ 43 & 1 & 0 & 3.096126 & -3.068402 & -1.309835 \\ 44 & 8 & 0 & 2.742593 & 0.886046 & 0.312824 \\ 45 & 8 & 0 & 4.985498 & -2.32384 & -0.835522 \\ 46 & 1 & 0 & 2.23708 & 0.090534 & -1.531011 \\ 47 & 1 & 0 & 2.669 & -1.80929 & 0.82394 \\ 48 & 6 & 0 & 6.336741 & 0.140519 & -0.167966 \\ 49 & 6 & 0 & 6.88125 & 1.370528 & 0.19425 \\ 50 & 1 & 0 & 7.958842 & 1.504231 & 0.163775 \\ 51 & 6 & 0 & 6.022118 & 2.403384 & 0.588634 \\ 52 & 6 & 0 & 4.627246 & 2.248145 & 0.618379 \\ 53 & 1 & 0 & 3.987427 & 3.073924 & 0.906049 \\ 54 & 8 & 0 & 6.49554 & 3.624554 & 0.95077 \\ 55 & 1 & 0 & 7.46427 & 3.641343 & 0.890502 \\ 56 & 8 & 0 & 7.163703 & -0.848175 & -0.540695 \\ 57 & 1 & 0 & 6.612712 & -1.641391 & -0.763089 \\ -------------------- & -----------19\end{array}$


<smiles>O=C1c2c(O)cc(O)cc2O[C@@H](c2ccc(O)c(O)c2)[C@@H]1O</smiles>

TAX

\begin{tabular}{|c|c|c|c|c|c|c|}
\hline \multirow{3}{*}{$\begin{array}{l}\text { Center } \\
\text { Number }\end{array}$} & \multirow{3}{*}{$\begin{array}{l}\text { Atomic } \\
\text { Number }\end{array}$} & \multicolumn{3}{|c|}{ 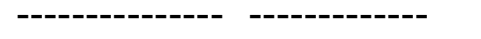 } & ------------ & \\
\hline & & Atomic & & \multicolumn{2}{|c|}{ Coordinates (Angstroms) } & \\
\hline & & Type & & $\mathrm{X}$ & $\mathrm{Y}$ & Z \\
\hline 1 & 6 & & 0 & 4571406 & -051853 & 0.1622 \\
\hline 2 & 6 & & 0 & 3.782624 & 0.618503 & 0.004122 \\
\hline 3 & 6 & & 0 & 2.362534 & 0.50655 & -0.071378 \\
\hline 4 & 6 & & 0 & 1.773736 & -0.781981 & 0.053409 \\
\hline 5 & 6 & & 0 & 2.553251 & -1.917202 & 0.211877 \\
\hline 6 & 6 & & 0 & 3.947979 & -1.76919 & 0.256114 \\
\hline 7 & 1 & & 0 & 5.651672 & -0.413977 & 0.209017 \\
\hline 8 & 1 & & 0 & 2.0984 & -2.895189 & 0.315491 \\
\hline 9 & 8 & & 0 & 4.383952 & 1.815169 & -0.092765 \\
\hline 10 & 1 & & 0 & 3.679798 & 2.503838 & -0.200929 \\
\hline 11 & 8 & & 0 & 4.667076 & -2.912119 & 0.413364 \\
\hline 12 & 1 & & 0 & 5.615947 & -2.709933 & 0.445486 \\
\hline 13 & 8 & & 0 & 0.425944 & -0.948874 & 0.006002 \\
\hline 14 & 6 & & 0 & 1.532242 & 1.649691 & -0.32105 \\
\hline 15 & 8 & & 0 & 1.94847 & 2.824629 & -0.377608 \\
\hline 16 & 6 & & 0 & 0.047129 & 1.386984 & -0.551793 \\
\hline 17 & 1 & & 0 & -0.081112 & 1.112904 & -1.614434 \\
\hline 18 & 8 & & 0 & -0.710824 & 2.532238 & -0.238048 \\
\hline 19 & 1 & & 0 & -0.09608 & 3.28942 & -0.301647 \\
\hline 20 & 6 & & 0 & -0.408179 & 0.194836 & 0.31009 \\
\hline 21 & 1 & & 0 & -0.250228 & 0.460357 & 1.365442 \\
\hline 22 & 6 & & 0 & -1.848317 & -0.199366 & 0.087597 \\
\hline 23 & 6 & & 0 & -2.215878 & -1.117973 & -0.901629 \\
\hline 24 & 6 & & 0 & -2.848042 & 0.405684 & 0.862554 \\
\hline 25 & 6 & & 0 & -3.560733 & -1.430697 & -1.116106 \\
\hline 26 & 1 & & 0 & -1.451048 & -1.6062 & -1.496462 \\
\hline 27 & 6 & & 0 & -4.185936 & 0.104538 & 0.637116 \\
\hline 28 & 1 & & 0 & -2.579924 & 1.130442 & 1.628797 \\
\hline 29 & 6 & & 0 & -4.553244 & -0.821198 & -0.352481 \\
\hline 30 & 8 & & 0 & -5.237051 & 0.65356 & 1.343995 \\
\hline 31 & 8 & & 0 & -5.866029 & -1.128894 & -0.567536 \\
\hline 32 & 1 & & 0 & -3.85502 & -2.149013 & -1.875481 \\
\hline
\end{tabular}




$\begin{array}{rrrrrr}33 & 1 & 0 & -6.41089 & -0.605627 & 0.045472 \\ 34 & 1 & 0 & -4.917237 & 1.3334 & 1.956182 \\ ------ & ------------~------------~ & ---------------- & ----------- & ----------\end{array}$




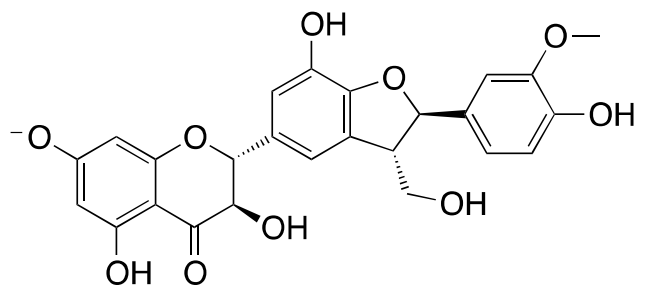

$\operatorname{SILYC}_{(-\mathrm{H})}{ }^{-1}$

\begin{tabular}{|c|c|c|c|c|c|}
\hline \multirow{2}{*}{$\begin{array}{l}\text { Center } \\
\text { Number }\end{array}$} & \multirow{2}{*}{$\begin{array}{l}\text { Atomic } \\
\text { Number }\end{array}$} & \multirow{2}{*}{$\begin{array}{l}\text { Atomic } \\
\text { Type }\end{array}$} & \multicolumn{3}{|c|}{ Coordinates (Angstroms) } \\
\hline & & & $\mathrm{X}$ & $\mathrm{Y}$ & $\mathrm{Z}$ \\
\hline 1 & 6 & 0 & -7.67279 & -0.227851 & -0.174194 \\
\hline 2 & 6 & 0 & -6.799962 & 0.835678 & 0.011342 \\
\hline 3 & 6 & 0 & -5.399903 & 0.609415 & 0.087309 \\
\hline 4 & 6 & 0 & -4.915294 & -0.714471 & -0.066352 \\
\hline 5 & 6 & 0 & -5.779555 & -1.777302 & -0.249445 \\
\hline 6 & 6 & 0 & -7.152488 & -1.517711 & -0.293455 \\
\hline 7 & 1 & 0 & -8.743463 & -0.033586 & -0.221252 \\
\hline 8 & 1 & 0 & -5.401415 & -2.787916 & -0.374526 \\
\hline 9 & 8 & 0 & -7.307838 & 2.062951 & 0.134261 \\
\hline 10 & 1 & 0 & -6.562693 & 2.698634 & 0.261403 \\
\hline 11 & 8 & 0 & -7.957101 & -2.585524 & -0.476293 \\
\hline 12 & 8 & 0 & -3.594344 & -0.984006 & -0.027946 \\
\hline 13 & 6 & 0 & -4.485119 & 1.670937 & 0.383964 \\
\hline 14 & 8 & 0 & -4.795157 & 2.868778 & 0.471772 \\
\hline 15 & 6 & 0 & -3.046861 & 1.26744 & 0.628432 \\
\hline 16 & 1 & 0 & -2.984524 & 0.90493 & 1.676987 \\
\hline 17 & 8 & 0 & -2.185746 & 2.340191 & 0.411481 \\
\hline 18 & 1 & 0 & -2.730586 & 3.14632 & 0.481116 \\
\hline 19 & 6 & 0 & -2.686816 & 0.101802 & -0.288071 \\
\hline 20 & 1 & 0 & -2.828075 & 0.428975 & -1.333726 \\
\hline 21 & 6 & 0 & -1.285158 & -0.388561 & -0.095121 \\
\hline 22 & 6 & 0 & -0.960591 & -1.129917 & 1.04602 \\
\hline 23 & 6 & 0 & -0.304434 & -0.045836 & -1.029734 \\
\hline 24 & 6 & 0 & 0.346139 & -1.543089 & 1.276594 \\
\hline 25 & 1 & 0 & -1.728321 & -1.415386 & 1.764313 \\
\hline 26 & 6 & 0 & 1.001062 & -0.45759 & -0.800703 \\
\hline 27 & 1 & 0 & -0.553612 & 0.552223 & -1.905033 \\
\hline 28 & 6 & 0 & 1.304762 & -1.182308 & 0.337963 \\
\hline 29 & 8 & 0 & 2.626235 & -1.502203 & 0.46469 \\
\hline 30 & 6 & 0 & 3.232981 & -1.229491 & -0.820636 \\
\hline 31 & 6 & 0 & 2.29708 & -0.207516 & -1.526684 \\
\hline 32 & 1 & 0 & 2.235179 & -0.44622 & -2.599082 \\
\hline
\end{tabular}




$\begin{array}{rrrrrr}33 & 6 & 0 & 2.730732 & 1.24734 & -1.416563 \\ 34 & 1 & 0 & 3.758809 & 1.368293 & -1.798446 \\ 35 & 1 & 0 & 2.720471 & 1.562816 & -0.357521 \\ 36 & 8 & 0 & 1.812755 & 1.997516 & -2.185829 \\ 37 & 1 & 0 & 1.94376 & 2.939336 & -2.017848 \\ 38 & 6 & 0 & 4.662096 & -0.81719 & -0.645731 \\ 39 & 6 & 0 & 5.080896 & -0.138913 & 0.504549 \\ 40 & 6 & 0 & 5.568339 & -1.044992 & -1.676224 \\ 41 & 6 & 0 & 6.391245 & 0.305619 & 0.605425 \\ 42 & 1 & 0 & 4.367456 & 0.019311 & 1.310454 \\ 43 & 6 & 0 & 6.882066 & -0.588963 & -1.581206 \\ 44 & 1 & 0 & 5.24815 & -1.583761 & -2.569226 \\ 45 & 6 & 0 & 7.298445 & 0.08652 & -0.445263 \\ 46 & 1 & 0 & 7.604408 & -0.759714 & -2.37697 \\ 47 & 1 & 0 & 3.191601 & -2.172125 & -1.392145 \\ 48 & 8 & 0 & 8.578259 & 0.519346 & -0.346764 \\ 49 & 1 & 0 & 8.687751 & 0.952015 & 0.516449 \\ 50 & 8 & 0 & 0.655481 & -2.267313 & 2.384283 \\ 51 & 1 & 0 & 1.591444 & -2.518612 & 2.341975 \\ 52 & 8 & 0 & 6.929401 & 0.963312 & 1.673514 \\ 53 & 6 & 0 & 6.09518 & 1.215825 & 2.783028 \\ 54 & 1 & 0 & 5.242614 & 1.850019 & 2.500173 \\ 55 & 1 & 0 & 5.723304 & 0.27695 & 3.217632 \\ 56 & 1 & 0 & 6.709882 & 1.739848 & 3.518458 \\ ------ & -------------------------------- & ------------ & -----------\end{array}$




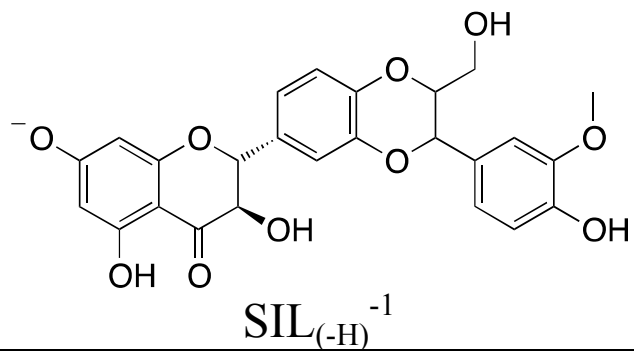

\begin{tabular}{|c|c|c|c|c|c|}
\hline \multirow{3}{*}{$\begin{array}{l}\text { Center } \\
\text { Number }\end{array}$} & \multirow{3}{*}{$\begin{array}{l}\text { Atomic } \\
\text { Number }\end{array}$} & ------------ & \multicolumn{3}{|c|}{---------- } \\
\hline & & \multirow{2}{*}{$\begin{array}{l}\text { Atomic } \\
\text { Type }\end{array}$} & \multicolumn{3}{|c|}{ Coordinates (Angstroms) } \\
\hline & & & $\mathrm{X}$ & Y & $\mathrm{Z}$ \\
\hline 1 & 6 & 0 & -7.784578 & 0.003911 & -1.131784 \\
\hline 2 & 6 & 0 & -6.980316 & 0.93962 & -0.494836 \\
\hline 3 & 6 & 0 & -5.635377 & 0.623911 & -0.165241 \\
\hline 4 & 6 & 0 & -5.127715 & -0.64825 & -0.530555 \\
\hline 5 & 6 & 0 & -5.923447 & -1.584366 & -1.162541 \\
\hline 6 & 6 & 0 & -7.249166 & -1.245282 & -1.449098 \\
\hline 7 & 1 & 0 & -8.815329 & 0.264189 & -1.368645 \\
\hline 8 & 1 & 0 & -5.525206 & -2.553644 & -1.448722 \\
\hline 9 & 8 & 0 & -7.504986 & 2.12613 & -0.187098 \\
\hline 10 & 1 & 0 & -6.810855 & 2.669248 & 0.258971 \\
\hline 11 & 8 & 0 & -7.985885 & -2.189934 & -2.069454 \\
\hline 12 & 8 & 0 & -3.850975 & -0.994413 & -0.264099 \\
\hline 13 & 6 & 0 & -4.81405 & 1.530462 & 0.5797 \\
\hline 14 & 8 & 0 & -5.138257 & 2.686396 & 0.890371 \\
\hline 15 & 6 & 0 & -3.467195 & 1.002828 & 1.026778 \\
\hline 16 & 1 & 0 & -3.638124 & 0.4014 & 1.945625 \\
\hline 17 & 8 & 0 & -2.576646 & 2.047305 & 1.262461 \\
\hline 18 & 1 & 0 & -3.119382 & 2.842318 & 1.419453 \\
\hline 19 & 6 & 0 & -2.909195 & 0.06654 & -0.04123 \\
\hline 20 & 1 & 0 & -2.801955 & 0.641209 & -0.97924 \\
\hline 21 & 6 & 0 & -1.594111 & -0.53899 & 0.342479 \\
\hline 22 & 6 & 0 & -1.530689 & -1.633479 & 1.209322 \\
\hline 23 & 6 & 0 & -0.415483 & 0.028772 & -0.129137 \\
\hline 24 & 6 & 0 & -0.301616 & -2.143083 & 1.603777 \\
\hline 25 & 1 & 0 & -2.449117 & -2.095838 & 1.567569 \\
\hline 26 & 6 & 0 & 0.815992 & -0.479503 & 0.264194 \\
\hline 27 & 1 & 0 & -0.43105 & 0.88381 & -0.804675 \\
\hline 28 & 6 & 0 & 0.877873 & -1.561939 & 1.146492 \\
\hline 29 & 8 & 0 & 1.951015 & 0.111399 & -0.213606 \\
\hline 30 & 8 & 0 & 2.067715 & -2.084769 & 1.558304 \\
\hline 31 & 6 & 0 & 3.212967 & -1.27162 & 1.308073 \\
\hline 32 & 1 & 0 & 4.073392 & -1.952631 & 1.362604 \\
\hline
\end{tabular}




$\begin{array}{rrrrrr}33 & 6 & 0 & 3.114601 & -0.696518 & -0.10864 \\ 34 & 1 & 0 & 3.00873 & -1.552804 & -0.800867 \\ 35 & 6 & 0 & 3.371437 & -0.225777 & 2.411626 \\ 36 & 1 & 0 & 3.067824 & -0.693305 & 3.35584 \\ 37 & 1 & 0 & 2.701945 & 0.633291 & 2.235596 \\ 38 & 8 & 0 & 4.709878 & 0.18229 & 2.575434 \\ 39 & 1 & 0 & 4.950415 & 0.775415 & 1.84651 \\ 40 & 6 & 0 & 4.31329 & 0.12404 & -0.491568 \\ 41 & 6 & 0 & 4.294891 & 1.514822 & -0.46282 \\ 42 & 6 & 0 & 5.499389 & -0.543906 & -0.82589 \\ 43 & 6 & 0 & 5.452108 & 2.241486 & -0.752378 \\ 44 & 1 & 0 & 3.370563 & 2.035611 & -0.221934 \\ 45 & 6 & 0 & 6.648558 & 0.177505 & -1.109643 \\ 46 & 1 & 0 & 5.5102 & -1.6335 & -0.854841 \\ 47 & 6 & 0 & 6.62832 & 1.583707 & -1.069898 \\ 48 & 8 & 0 & 7.746432 & 2.291674 & -1.350532 \\ 49 & 1 & 0 & -0.228061 & -2.993126 & 2.279828 \\ 50 & 1 & 0 & 5.454125 & 3.329675 & -0.738458 \\ 51 & 1 & 0 & 8.464541 & 1.666586 & -1.545536 \\ 52 & 8 & 0 & 7.859557 & -0.34906 & -1.445286 \\ 53 & 6 & 0 & 7.984926 & -1.754202 & -1.480281 \\ 54 & 1 & 0 & 9.021916 & -1.964109 & -1.75201 \\ 55 & 1 & 0 & 7.769022 & -2.192871 & -0.495616 \\ 56 & 1 & 0 & 7.315152 & -2.191186 & -2.23481 \\ ------------------------------------ & --------- & --------15\end{array}$




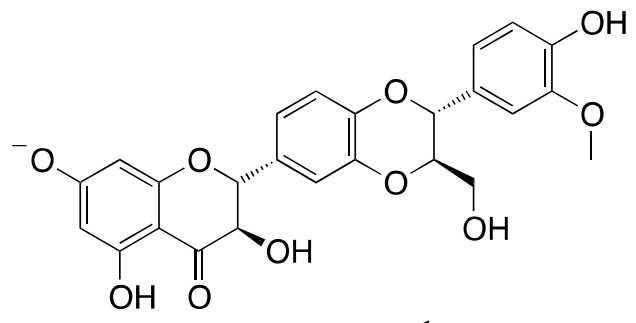

$\operatorname{ISOSIL}_{(-\mathrm{H})}{ }^{-1}$

\begin{tabular}{|c|c|c|c|c|c|}
\hline \multirow{2}{*}{$\begin{array}{l}\text { Center } \\
\text { Number }\end{array}$} & \multirow{2}{*}{$\begin{array}{l}\text { Atomic } \\
\text { Number }\end{array}$} & \multirow{2}{*}{$\begin{array}{l}\text { Atomic } \\
\text { Type }\end{array}$} & \multicolumn{3}{|c|}{ Coordinates (Angstroms) } \\
\hline & & & $\mathrm{X}$ & $\mathrm{Y}$ & Z \\
\hline 1 & 6 & 0 & -7.946901 & 1.119206 & 0.314799 \\
\hline 2 & 6 & 0 & -7.315362 & -0.066754 & 0.046639 \\
\hline 3 & 6 & 0 & -5.879248 & -0.138122 & -0.126322 \\
\hline 4 & 6 & 0 & -5.145638 & 1.088596 & 0.037977 \\
\hline 5 & 6 & 0 & -5.755822 & 2.276545 & 0.312129 \\
\hline 6 & 6 & 0 & -7.209245 & 2.364386 & 0.453734 \\
\hline 7 & 1 & 0 & -9.02617 & 1.147949 & 0.432158 \\
\hline 8 & 1 & 0 & -5.171735 & 3.182196 & 0.444124 \\
\hline 9 & 8 & 0 & -8.038489 & -1.215143 & -0.081223 \\
\hline 10 & 1 & 0 & -7.396048 & -1.943532 & -0.277486 \\
\hline 11 & 8 & 0 & -7.776525 & 3.4582 & 0.699132 \\
\hline 12 & 8 & 0 & -3.766697 & 1.081223 & -0.112167 \\
\hline 13 & 6 & 0 & -5.219079 & -1.327244 & -0.463997 \\
\hline 14 & 8 & 0 & -5.750774 & -2.479074 & -0.57883 \\
\hline 15 & 6 & 0 & -3.708177 & -1.256575 & -0.713543 \\
\hline 16 & 1 & 0 & -3.534697 & -0.990113 & -1.77056 \\
\hline 17 & 8 & 0 & -3.132227 & -2.521406 & -0.4347 \\
\hline 18 & 1 & 0 & -3.905098 & -3.128448 & -0.454835 \\
\hline 19 & 6 & 0 & -3.095224 & -0.151576 & 0.164121 \\
\hline 20 & 1 & 0 & -3.263783 & -0.427934 & 1.216674 \\
\hline 21 & 6 & 0 & -1.612358 & 0.056849 & -0.072606 \\
\hline 22 & 6 & 0 & -1.132838 & 1.128303 & -0.841012 \\
\hline 23 & 6 & 0 & -0.695709 & -0.851503 & 0.467779 \\
\hline 24 & 6 & 0 & 0.234044 & 1.276737 & -1.077942 \\
\hline 25 & 1 & 0 & -1.835906 & 1.848205 & -1.244278 \\
\hline 26 & 6 & 0 & 0.669608 & -0.699582 & 0.235591 \\
\hline 27 & 1 & 0 & -1.036311 & -1.696582 & 1.057815 \\
\hline 28 & 6 & 0 & 1.141433 & 0.359669 & -0.548553 \\
\hline 29 & 8 & 0 & 1.542374 & -1.627254 & 0.77652 \\
\hline 30 & 8 & 0 & 2.490505 & 0.535936 & -0.798532 \\
\hline 31 & 6 & 0 & 3.301104 & -0.62364 & -0.563903 \\
\hline 32 & 6 & 0 & 2.9184 & -1.228387 & 0.800647 \\
\hline
\end{tabular}




$\begin{array}{rrrrrr}33 & 1 & 0 & 3.051961 & -0.463092 & 1.580143 \\ 34 & 1 & 0 & 0.615826 & 2.100516 & -1.674972 \\ 35 & 6 & 0 & 3.695601 & -2.487455 & 1.174442 \\ 36 & 1 & 0 & 4.752958 & -2.250427 & 1.314058 \\ 37 & 1 & 0 & 3.606522 & -3.225978 & 0.36163 \\ 38 & 8 & 0 & 3.238323 & -3.035607 & 2.402886 \\ 39 & 6 & 0 & 4.750303 & -0.210276 & -0.653741 \\ 40 & 6 & 0 & 5.658108 & -0.954264 & -1.412212 \\ 41 & 6 & 0 & 5.220573 & 0.907838 & 0.050121 \\ 42 & 6 & 0 & 7.007284 & -0.594118 & -1.461121 \\ 43 & 1 & 0 & 5.31642 & -1.817687 & -1.9779 \\ 44 & 6 & 0 & 6.562923 & 1.280247 & 0.008489 \\ 45 & 1 & 0 & 4.537022 & 1.527141 & 0.622929 \\ 46 & 6 & 0 & 7.467646 & 0.522723 & -0.762045 \\ 47 & 1 & 0 & 7.703936 & -1.1743 & -2.06502 \\ 48 & 8 & 0 & 8.777359 & 0.935174 & -0.80123 \\ 49 & 1 & 0 & 9.278558 & 0.353941 & -1.393937 \\ 50 & 8 & 0 & 6.955486 & 2.428026 & 0.654643 \\ 51 & 6 & 0 & 7.835773 & 2.253492 & 1.771066 \\ 52 & 1 & 0 & 8.006349 & 3.254031 & 2.174876 \\ 53 & 1 & 0 & 7.362576 & 1.62534 & 2.537704 \\ 54 & 1 & 0 & 8.788828 & 1.811863 & 1.462102 \\ 55 & 1 & 0 & 2.270949 & -3.111689 & 2.338852 \\ 56 & 1 & 0 & 3.079979 & -1.372563 & -1.339303 \\ ------------------------------- & ----------- & ----------\end{array}$




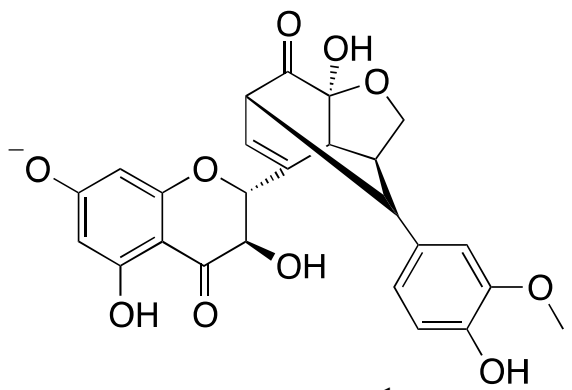

$\operatorname{SILYD}_{(-\mathrm{H})}{ }^{-1}$

\begin{tabular}{|c|c|c|c|c|c|}
\hline \multirow{2}{*}{$\begin{array}{l}------ \\
\text { Center } \\
\text { Number }\end{array}$} & \multirow{2}{*}{$\begin{array}{l}\text { Atomic } \\
\text { Number }\end{array}$} & \multirow{2}{*}{$\begin{array}{l}\text { Atomic } \\
\text { Type }\end{array}$} & \multicolumn{3}{|c|}{ Coordinates (Angstroms) } \\
\hline & & & $\mathrm{X}$ & $\mathrm{Y}$ & $\mathrm{Z}$ \\
\hline 1 & 6 & 0 & -1.117444 & -1.876957 & 0.401274 \\
\hline 2 & 8 & 0 & -2.335694 & -2.27838 & -0.272913 \\
\hline 3 & 6 & 0 & -2.415849 & -1.582913 & -1.530402 \\
\hline 4 & 6 & 0 & -1.449503 & -0.406995 & -1.393053 \\
\hline 5 & 6 & 0 & -0.274076 & -1.123276 & -0.652335 \\
\hline 6 & 1 & 0 & -2.095582 & -2.255269 & -2.339037 \\
\hline 7 & 1 & 0 & -3.456348 & -1.294603 & -1.698708 \\
\hline 8 & 1 & 0 & -1.150294 & -0.022706 & -2.373322 \\
\hline 9 & 1 & 0 & 0.235315 & -1.838806 & -1.299881 \\
\hline 10 & 6 & 0 & 0.681801 & -0.16617 & 0.027709 \\
\hline 11 & 6 & 0 & 0.093508 & 0.681584 & 0.889597 \\
\hline 12 & 1 & 0 & 0.637353 & 1.4109 & 1.478585 \\
\hline 13 & 6 & 0 & -1.421495 & 0.571123 & 0.971254 \\
\hline 14 & 1 & 0 & -1.865786 & 1.277687 & 1.675286 \\
\hline 15 & 6 & 0 & -1.587148 & -0.857124 & 1.460999 \\
\hline 16 & 8 & 0 & -0.485902 & -2.959798 & 0.975517 \\
\hline 17 & 1 & 0 & -0.903515 & -3.103028 & 1.846156 \\
\hline 18 & 8 & 0 & -1.989559 & -1.208163 & 2.55849 \\
\hline 19 & 6 & 0 & -1.971831 & 0.772 & -0.48853 \\
\hline 20 & 1 & 0 & -1.462896 & 1.67348 & -0.846919 \\
\hline 21 & 6 & 0 & -3.462301 & 1.056757 & -0.573274 \\
\hline 22 & 6 & 0 & -3.933047 & 2.023988 & -1.469034 \\
\hline 23 & 6 & 0 & -4.401588 & 0.378091 & 0.226367 \\
\hline 24 & 6 & 0 & -5.298673 & 2.310983 & -1.582802 \\
\hline 25 & 1 & 0 & -3.225101 & 2.571538 & -2.087207 \\
\hline 26 & 6 & 0 & -5.760262 & 0.66341 & 0.120626 \\
\hline 27 & 1 & 0 & -4.067986 & -0.376734 & 0.927912 \\
\hline 28 & 6 & 0 & -6.217539 & 1.634427 & -0.790401 \\
\hline 29 & 1 & 0 & -5.659093 & 3.065084 & -2.276717 \\
\hline 30 & 8 & 0 & -7.555194 & 1.91296 & -0.888953 \\
\hline
\end{tabular}




$\begin{array}{rrrrrr}31 & 1 & 0 & -8.019227 & 1.341528 & -0.252115 \\ 32 & 8 & 0 & -6.761949 & 0.065253 & 0.852194 \\ 33 & 6 & 0 & -6.399386 & -0.937755 & 1.799448 \\ 34 & 1 & 0 & -5.737525 & -0.528518 & 2.571919 \\ 35 & 1 & 0 & -7.335349 & -1.268709 & 2.253169 \\ 36 & 1 & 0 & -5.906319 & -1.784126 & 1.306636 \\ 37 & 6 & 0 & 2.15271 & -0.141948 & -0.312452 \\ 38 & 6 & 0 & 2.889225 & -1.489203 & -0.205473 \\ 39 & 6 & 0 & 4.388758 & -1.273851 & -0.456943 \\ 40 & 6 & 0 & 4.127516 & 1.025821 & 0.396478 \\ 41 & 6 & 0 & 4.947731 & -0.03411 & -0.122714 \\ 42 & 8 & 0 & 2.406685 & -2.416441 & -1.16619 \\ 43 & 1 & 0 & 3.223078 & -2.877044 & -1.463119 \\ 44 & 8 & 0 & 2.766818 & 0.809557 & 0.557045 \\ 45 & 8 & 0 & 5.004508 & -2.259956 & -0.97715 \\ 46 & 1 & 0 & 2.261542 & 0.196667 & -1.358875 \\ 47 & 1 & 0 & 2.747827 & -1.89227 & 0.8109 \\ 48 & 6 & 0 & 6.361925 & 0.247144 & -0.265055 \\ 49 & 6 & 0 & 6.893471 & 1.452676 & 0.111061 \\ 50 & 1 & 0 & 7.957906 & 1.637163 & 0.000517 \\ 51 & 6 & 0 & 6.067872 & 2.515276 & 0.661354 \\ 52 & 6 & 0 & 4.636048 & 2.233319 & 0.77093 \\ 53 & 1 & 0 & 3.987019 & 3.016353 & 1.151095 \\ 54 & 8 & 0 & 6.542453 & 3.624356 & 1.010364 \\ 55 & 8 & 0 & 7.165909 & -0.726959 & -0.775385 \\ 56 & 1 & 0 & 6.58783 & -1.5058 & -0.978199 \\ ------------------------------------- & ----------- & -----------\end{array}$


<smiles>COc1cc(O)c2c(c1)O[C@H](c1ccc(O)c(O)c1)C(O)C2=O</smiles>

$\operatorname{TAX}_{(-\mathrm{H})}{ }^{-1}$

\begin{tabular}{|c|c|c|c|c|c|}
\hline \multirow{2}{*}{$\begin{array}{l}\text { Center } \\
\text { Number }\end{array}$} & \multirow{2}{*}{$\begin{array}{l}\text { Atomic } \\
\text { Number }\end{array}$} & \multirow{2}{*}{$\begin{array}{l}\text { Atomic } \\
\text { Type }\end{array}$} & \multicolumn{3}{|c|}{ Coordinates (Angstroms) } \\
\hline & & & $\mathrm{X}$ & $\mathrm{Y}$ & $\mathrm{Z}$ \\
\hline 1 & 6 & 0 & 4.614053 & -0.5333 & 0.17781 \\
\hline 2 & 6 & 0 & 3.831273 & 0.578925 & 0.013115 \\
\hline 3 & 6 & 0 & 2.391155 & 0.480315 & -0.105838 \\
\hline 4 & 6 & 0 & 1.820759 & -0.836624 & -0.002749 \\
\hline 5 & 6 & 0 & 2.583136 & -1.953796 & 0.168058 \\
\hline 6 & 6 & 0 & 4.040936 & -1.867489 & 0.25546 \\
\hline 7 & 1 & 0 & 5.692672 & -0.434426 & 0.256904 \\
\hline 8 & 1 & 0 & 2.121036 & -2.932345 & 0.255846 \\
\hline 9 & 8 & 0 & 4.401621 & 1.814855 & -0.058824 \\
\hline 10 & 1 & 0 & 3.666681 & 2.467788 & -0.182583 \\
\hline 11 & 8 & 0 & 4.748024 & -2.894793 & 0.406782 \\
\hline 12 & 8 & 0 & 0.446831 & -0.990521 & -0.107389 \\
\hline 13 & 6 & 0 & 1.575984 & 1.596187 & -0.333929 \\
\hline 14 & 8 & 0 & 1.957183 & 2.811212 & -0.38683 \\
\hline 15 & 6 & 0 & 0.073786 & 1.356493 & -0.525759 \\
\hline 16 & 1 & 0 & -0.122513 & 1.150819 & -1.591837 \\
\hline 17 & 8 & 0 & -0.628015 & 2.52436 & -0.124568 \\
\hline 18 & 1 & 0 & 0.070433 & 3.216893 & -0.139286 \\
\hline 19 & 6 & 0 & -0.357545 & 0.12392 & 0.288177 \\
\hline 20 & 1 & 0 & -0.166222 & 0.344451 & 1.350804 \\
\hline 21 & 6 & 0 & -1.815506 & -0.257152 & 0.106998 \\
\hline 22 & 6 & 0 & -2.203855 & -1.429358 & -0.551239 \\
\hline 23 & 6 & 0 & -2.81207 & 0.593549 & 0.611122 \\
\hline 24 & 6 & 0 & -3.558008 & -1.747273 & -0.709231 \\
\hline 25 & 1 & 0 & -1.441881 & -2.098578 & -0.933556 \\
\hline 26 & 6 & 0 & -4.154251 & 0.278786 & 0.443339 \\
\hline 27 & 1 & 0 & -2.527824 & 1.518496 & 1.107361 \\
\hline 28 & 6 & 0 & -4.541263 & -0.896051 & -0.215218 \\
\hline 29 & 8 & 0 & -5.195214 & 1.074015 & 0.90424 \\
\hline 30 & 8 & 0 & -5.872332 & -1.203946 & -0.371471 \\
\hline 31 & 1 & 0 & -3.862328 & -2.658073 & -1.21773 \\
\hline 32 & 1 & 0 & -6.390794 & -0.481672 & 0.021994 \\
\hline
\end{tabular}




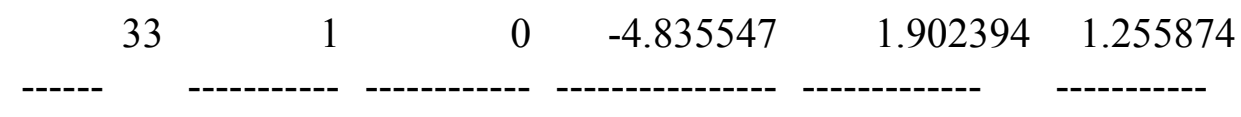

